\title{
INTERIOR AND BOUNDARY STABILIZATION OF NAVIER-STOKES EQUATIONS
}

\author{
Roberto Triggiani* \\ University of Virginia \\ Charlottesville, VA 22904 USA \\ rt7u@virginia.edu
}

Abstract We report on very recent work on the stabilization of the steady-state solutions to Navier-Stokes equations on an open bounded domain $\Omega \subset$ $R^{d}, d=2,3$, by either interior, or else boundary control.

More precisely, as to the interior case, we obtain that the steadystate solutions to Navier-Stokes equations on $\Omega \subset R^{d}, d=2,3$, with no-slip boundary conditions, are locally exponentially stabilizable by a finite-dimensional feedback controller with support in an arbitrary open subset $\omega \subset \Omega$ of positive measure. The (finite) dimension of the feedback controller is minimal and is related to the largest algebraic multiplicity of the unstable eigenvalues of the linearized equation.

Second, as to the boundary case, we obtain that the steady-state solutions to Navier-Stokes equations on a bounded domain $\Omega \subset R^{d}$, $d=2,3$, are locally exponentially stabilizable by a boundary closedloop feedback controller, acting on the boundary $\partial \Omega$, in the Dirichlet boundary conditions. If $d=3$, the non-linearity imposes and dictates the requirement that stabilization must occur in the space $\left(H^{\frac{3}{2}+\epsilon}(\Omega)\right)^{3}$, $\epsilon>0$, a high topological level. A first implication thereof is that, for $d=3$, the boundary feedback stabilizing controller must be infinite dimensional. Moreover, it generally acts on the entire boundary $\partial \Omega$. Instead, for $d=2$, where the topological level for stabilization is $\left(H^{\frac{3}{2}-\epsilon}(\Omega)\right)^{2}$, the boundary feedback stabilizing controller can be chosen to act on an arbitrarily small portion of the boundary. Moreover, still for $d=2$, it may even be finite dimensional, and this occurs if the linearized operator is diagonalizable over its finite-dimensional unstable subspace.

Keywords: Internal stabilization, boundary stabilization, Navier-Stokes Equations. 
We hereby report on recent joint work on the stabilization of steadystate solutions to Navier-Stokes equations on an open bounded domain $\Omega \subset R^{d}, d=2,3$, by either interior feedback control or else boundary feedback control. The case of interior control is taken from the joint work with V. Barbu in [4]. The case of boundary control is taken from the joint work with V. Barbu and I. Lasiecka in [3]. To enhance readability, we provide independent accounts of each case.

\section{Part I: Interior Control [4]}

\section{Introduction}

The controlled N-S equations. Consider the controlled NavierStokes equations (see [6, p. 45], [13, p. 253] for the uncontrolled case $u \equiv 0)$ with the non-slip Dirichlet B.C.:

$$
\begin{aligned}
y_{t}(x, t)-\nu \Delta y(x, t)+(y \cdot \nabla) y(x, t) & \\
=m(x) u(x, t)+f_{e}(x)+\nabla p(x, t) \text { in } Q=\Omega \times(0, \infty), & \\
\nabla \cdot y=0 & \text { in } Q ; \\
y=0 & \text { on } \Sigma=\partial \Omega \times(0, \infty) ; \\
y(x, 0)=y_{0}(x) & \text { in } \Omega .
\end{aligned}
$$

Here, $\Omega$ is an open smooth bounded domain of $R^{d}, d=2,3 ; m$ is the characteristic function of an open smooth subset $\omega \subset \Omega$ of positive measure; $u$ is the control input; and $y=\left(y_{1}, y_{2}, \ldots, y_{d}\right)$ is the state (velocity) of the system. The function $v=m u$ can be viewed itself as an internal controller with support in $Q_{\omega}=\omega \times(0, \infty)$. The functions $y_{0}, f_{e} \in\left(L^{2}(\Omega)\right)^{d}$ are given, the latter being a body force, while $p$ is the unknown pressure.

Let $\left(y_{e}, p_{e}\right) \in\left(\left(H^{2}(\Omega)\right)^{d} \cap V\right) \times H^{1}(\Omega)$ be a steady-state solution to equation (1), i.e.,

$$
\begin{aligned}
& -\nu \Delta y_{e}+\left(y_{e} \cdot \nabla\right) y_{e}=f_{e}+\nabla p_{e} \text { in } \Omega ; \\
& \nabla \cdot y_{e}=0 \text { in } \Omega \text {; } \\
& y_{e}=0 \text { on } \partial \Omega \text {. }
\end{aligned}
$$

The steady-state solution is known to exist for $d=2,3,[6$, Theorem 7.3, p. 59]. Here [6, p. 9], [13, p. 18]

$$
\begin{aligned}
V & =\left\{y \in\left(H_{0}^{1}(\Omega)\right)^{d} ; \nabla \cdot y=0\right\}, \text { with norm }\|y\|_{V} \equiv\|y\| \\
& =\left\{\int_{\Omega}|\nabla y(x)|^{2} d \Omega\right\}^{\frac{1}{2}} .
\end{aligned}
$$


Literature. According to some recent results of O. Imanuvilov [9] (see also [1]) any such solution $y_{e}$ is locally exactly controllable on every interval $[0, T]$ with controller $u$ with support in $Q_{\omega}$. More precisely, if the distance $\left\|y_{e}-y_{0}\right\|_{H^{2}(\Omega)}$ is sufficiently small, then there is a solution $(y, p, u)$ to $(1)$ of appropriate regularity such that $y(T) \equiv y_{e}$. The steering control is open-loop and depends on the initial condition. Subsequently, paper [2] proved that any steady-state solution $y_{e}$ is locally exponentially stabilizable by means of an infinite-dimensional feedback controller, by using the controllability of the linear Stokes equation. In contrast, here we shall prove, via the state decomposition technique of [14], [15], and the first-order stabilization Riccati equation method developed in our previous work [2] (see also [5] still in the parabolic case, as well as [11] in the hyperbolic case), that any steady-state solution $y_{e}$ is locally exponentially stabilizable by a finite-dimensional closed-loop feedback controller of the form

$$
u=-\sum_{i=1}^{2 K}\left(R_{N}\left(y-y_{e}\right), \psi_{i}\right)_{\omega} \psi_{i},
$$

where $R_{N} \in \mathcal{L}\left(\mathcal{D}\left(A^{\frac{1}{4}}\right)\right) \cap \mathcal{L}\left(\mathcal{D}\left(A^{\frac{1}{2}}\right) ; H\right)$ is the solution of the algebraic Riccati equation (18) below associated with the linearized system (14) below and $\left\{\psi_{i}\right\}_{i=1}^{2 K}$ is an explicitly constructed (in (3.3.5) of [4]) system of functions related to the space of eigenfunctions corresponding to the unstable eigenvalues of such linearized system. Here $A$ is the Stokes operator defined by $(6) ; H$ the space in $(5)$; and $(\cdot, \cdot)_{\omega}$ is the scalar product in $\left(L^{2}(\omega)\right)^{d}$. The present closed-loop feedback stabilization result has two main features, besides being finite-dimensional:

(1) it is more precise and less restrictive concerning the vectors $y_{0}$ and $y_{e}$ than the open-loop version provided by the local exact controllability result established in [9], or the closed-loop stabilization in [2] (in that smallness of the distance between $y_{0}$ and $y_{e}$ is measured in the $\mathcal{D}\left(A^{\frac{1}{4}}\right)$ norm, i.e., the $\left(H^{\frac{1}{2}}(\Omega)\right)^{d}$-norm, see the set $\mathcal{V}_{\rho}$ in $(21)$ below, rather than in the $\left(H^{2}(\Omega)\right)^{d}$-norm, as recalled above, where $A$ is defined in (6).);

(2) it is independent of the Carleman inequality for the Stokes equation, which is necessary for the proof of local controllability.

There is a large literature on the stabilization problem of steadystate solutions to Navier-Stokes equations. Here we confine ourselves to mention only a few of the papers ([2], [7]) which are more related to this present work. We also refer to the recent paper of Fursikov [8] for a study of a boundary-rather than interior-problem for the N-S equations, which, however, does not pertain to the topic of feedback stabilization in the established sense, as in the present paper. 
Notation. Here we shall use the standard notation for the spaces of summable functions and Sobolev spaces on $\Omega$. In particular, $H^{s}(\Omega)$ is the Sobolev space of order $s$ with the norm denoted by $\|\cdot\|_{s}$. The following notation will be also used:

$$
\begin{gathered}
\nabla \cdot y=\operatorname{div} y,(y \cdot \nabla) y=y_{i} D_{i} y_{j}=y \cdot \nabla y_{j} \\
j=1, \ldots, d, D_{i}=\frac{\partial}{\partial x_{i}} \\
H=\left\{y \in\left(L^{2}(\Omega)\right)^{d} ; \nabla \cdot y=0, y \cdot n=0 \text { on } \partial \Omega\right\} \quad[6, \text { p. } 7] ; \\
H^{\perp}=\left\{y \in\left(L^{2}(\Omega)\right)^{d}: y=\nabla p, p \in H^{1}(\Omega)\right\},\left(L^{2}(\Omega)\right)^{d}=H+H^{\perp},
\end{gathered}
$$

$H^{\perp}$ being the orthogonal complement of $H$ in $\left(L^{2}(\Omega)\right)^{d}[13$, p. 15] with summation convention to be used throughout the paper, presently in $i=1, \ldots, d$, where $n$ is the outward normal to the boundary $\partial \Omega$ of $\Omega$. We shall denote by $P:\left(L^{2}(\Omega)\right)^{d} \rightarrow H$ the orthogonal Leray projector $[6$, p. 9$]$, and moreover $[6$, p. 31],

$$
A y=-P \Delta y, \quad \forall y \in \mathcal{D}(A)=\left(H^{2}(\Omega)\right)^{d} \cap V, \quad V=\mathcal{D}\left(A^{\frac{1}{2}}\right),
$$

which is a self-adjoint positive definite operator in $H$ with compact (resolvent) $A^{-1}$ on $H[6, \mathrm{p} .32]$. Accordingly, the fractional powers $A^{s}$, $0<s<1$, are well-defined [6, p. 33]. We have $V=\mathcal{D}\left(A^{\frac{1}{2}}\right)[6$, p. 33]. Furthermore, we define $B: V \rightarrow V^{\prime}$ by $[6$, p. 47, p. 54], [13, p. 162],

$$
B y=P[(y \cdot \nabla) y],(B y, w)=b(y, y, w), \quad \forall y, w \in V,
$$

where the trilinear form is defined by $[6$, p. 49], [13, p. 161]

$$
\begin{array}{r}
b(y, z, w)=\int_{\Omega} y_{i}\left(D_{i} z_{j}\right) w_{j} d x=\int_{\Omega}\langle y \cdot \nabla z, w\rangle_{R^{d}} d \Omega, \\
y, w \in H, z \in V .
\end{array}
$$

We shall denote by $(\cdot, \cdot)$ the scalar product in both $H$ and $\left(L^{2}(\Omega)\right)^{d}$. Similarly, we shall denote by the same symbol $|\cdot|$ the norm of both $\left(L^{2}(\Omega)\right)^{d}$ and $H$, and by $\|\cdot\|$ the norm of the space $V$ as defined in (3).

Preliminaries. In the notation introduced above, Eqn. (1) can be equivalently rewritten in abstract form as

$$
\frac{d y}{d t}+\nu A y+B y=P\left(m u+f_{e}\right) ; \quad y(0)=y_{0} \in H,
$$

since the procedure of applying $P$ to $(1)$ eliminates the pressure from the equations [6, p. 47], the orthogonal space $H^{\perp}$ to $H$ being made up of $\left(L^{2}(\Omega)^{d}\right.$-functions which are the gradients of $H^{1}(\Omega)$-functions by $(5)$. Moreover, $y \in H$ for (1) implies $P y_{t}=y_{t}$. 


\section{The Main Results}

Assumptions. (i) The boundary $\partial \Omega$ of $\Omega$ is a finite union of $d-1$ dimensional $C^{2}$-connected manifolds. Moreover, the boundary $\partial \omega$ of $\omega$ is of class $C^{2}$.

(ii) The steady-state solution $\left(y_{e}, p_{e}\right)$ defined in (2) belongs to $\left(\left(H^{2}(\Omega)\right)^{d} \cap V\right) \times H^{1}(\Omega)$, where we recall from (6) that then $y_{e} \in \mathcal{D}(A)$. [For $d=2,3$, this property is guaranteed by [6, Theorem 7.3 , p. 59] on $y_{e}$, for $f_{e} \in H$, followed by $\left[6\right.$, Theorem 3.11, p. 30] on $p_{e}$, for sufficiently smooth $\partial \Omega$.]

Preliminaries. The translated problem. By the substitutions $y \rightarrow$ $y_{e}+y, p \rightarrow p_{e}+p$, we are readily led via (1), (2) to the study of null stabilization of the equation

$$
\begin{array}{rlrl}
y_{t}-\nu \Delta y+(y \cdot \nabla) y+\left(y_{e} \cdot \nabla\right) y+(y \cdot \nabla) y_{e} & =m u+\nabla p & & \text { in } Q \\
\nabla \cdot y & =0 & & \text { in } Q \\
y & =0 & & \text { on } \Sigma \\
y(x, 0)=y^{0}(x)=y_{0}(x) & -y_{e}(x) . &
\end{array}
$$

By use of (5) on $H,(6),(7)$ on $A$ and $B$, we see that (10), after application of $P$, can be rewritten abstractly as

$$
\frac{d y}{d t}+\nu A y+A_{0} y+B y=P(m u), t>0 ; \quad y(0)=y^{0}
$$

(compare with (9) again $P y_{t}=y_{t}$, since $y \in H$ by (10)), where we have now introduced the operator $A_{0} \in \mathcal{L}(V ; H)$,

$$
A_{0} y=P\left(\left(y_{e} \cdot \nabla\right) y+(y \cdot \nabla) y_{e}\right), \quad \mathcal{D}\left(A_{0}\right)=V=\mathcal{D}\left(A^{\frac{1}{2}}\right)
$$

or equivalently, recalling (7),

$$
\left(A_{0} y, z\right)=b\left(y_{e}, y, z\right)+b\left(y, y_{e}, z\right), \quad \forall y \in V, z \in H .
$$

The operator $A_{0}$ in (12) is well-defined $H \supset V=\mathcal{D}\left(A_{0}\right) \rightarrow H$. This follows from the estimate

$$
\left|A_{0} y\right| \leq C_{1}\left\|y_{e}\right\|_{2}\|y\|, \quad \forall y \in V=\mathcal{D}\left(A_{0}\right)=\mathcal{D}\left(A^{\frac{1}{2}}\right),
$$

which is obtained directly by use of the definition (12b).

The linearized problem. Next, we consider the following linearized system of the translated model (10) or (11):

$$
\frac{d y}{d t}+\nu A y+A_{0} y=P(m u), t>0 ; \quad y(0)=y^{0} \in H .
$$


We have already noted below $(6)$ that the operator $-\nu A(\nu>0$, the viscosity coefficient) is negative self-adjoint and has compact resolvent on $H$. Thus, $-\nu A$ generates an analytic (self-adjoint) $C_{0}$-semigroup on $H$. It then follows from here and from $\mathcal{D}\left(A_{0}\right)=V=\mathcal{D}\left(A^{\frac{1}{2}}\right)$, as noted in (6) and in (13), that: the perturbed operator

$$
\mathcal{A}=-\left(\nu A+A_{0}\right) \text {, with domain } \mathcal{D}(\mathcal{A})=\mathcal{D}(A)=\left(H^{2}(\Omega)\right)^{d} \cap V
$$

likewise has compact resolvent and generates an analytic $C_{0}$-semigroup on $H$. This is well-known. It follows from the above claim that the operator $\mathcal{A}$ has a finite number $N$ of eigenvalues $\lambda_{j}$ with $\operatorname{Re} \lambda_{j} \geq 0$ (the unstable eigenvalues). The eigenvalues are repeated according to their algebraic multiplicity $\ell_{j}$. Let $\left\{\varphi_{j}\right\}_{j=1}^{N}$ be a corresponding system of generalized eigenfunctions, $\varphi_{j}=\varphi_{j}^{1}+i \varphi_{j}^{2}, j=1, \ldots, N$ of $\mathcal{A}$. (See $[10$, p. 41, 181].) More precisely, we shall denote by $M$ the number of distinct unstable eigenvalues, so that $\ell_{1}+\ell_{2}+\cdots+\ell_{M}=N$. In order to state our first result, we finally need to introduce the following finite-dimensional real spaces $X_{N}^{\alpha}, \alpha=1,2$ as well as the following natural number $K$ :

$$
X_{N}^{\alpha}=\operatorname{span}\left\{\varphi_{j}^{\alpha}\right\}_{j=1}^{N} ; \quad K=\max \left\{\ell_{j} ; 1 \leq j \leq M\right\} .
$$

Main results. Linearized problem (14). We first state the following feedback stabilization result for the linearized system (14).

THEOREM 1 Let $\epsilon>0$ be arbitrary but fixed, and let $\gamma_{0}=\left|\operatorname{Re} \lambda_{N+1}\right|-\epsilon$. Then, for each $\lambda, 0 \leq \lambda \leq \gamma_{0}$, there are functions $\left\{\psi_{i}\right\}_{i=1}^{K} \subset X_{N}^{1}$, $\left\{\psi_{i}\right\}_{i=K+1}^{2 K} \subset X_{N}^{2}$ and a linear self-adjoint operator $R_{N}: \mathcal{D}\left(R_{N}\right) \subset H \rightarrow$ $H$ such that for some constants $0<a_{1}<a_{2}<\infty$ and $C_{1}>0$, we have:

(i)

$$
a_{1}\left|A^{\frac{1}{4}} y\right|^{2} \leq\left(R_{N} y, y\right) \leq a_{2}\left|A^{\frac{1}{4}} y\right|^{2}, \quad \forall y \in \mathcal{D}\left(A^{\frac{1}{4}}\right),
$$

so that $\mathcal{D}\left(A^{\frac{1}{4}}\right) \subset \mathcal{D}\left(R_{N}^{\frac{1}{2}}\right)$;

(ii)

$$
\left|R_{N} y\right| \leq C_{1}\|y\|, \quad \forall y \in V=\mathcal{D}\left(A^{\frac{1}{2}}\right) ;
$$

(iii) $R_{N}$ satisfies the following algebraic Riccati equation:

$$
-\left((\mathcal{A}+\lambda) y, R_{N} y\right)+\frac{1}{2} \sum_{i=1}^{2 K}\left(\psi_{i}, R_{N} y\right)_{\omega}^{2}=\frac{1}{2}\left|A^{\frac{3}{4}} y\right|^{2}, \quad \forall y \in \mathcal{D}(A) .
$$

The vectors $\left\{\psi_{i}\right\}_{i=1}^{2 K}$ are explicitly constructed in (3.3.5) of Lemma 4 of [4]. Moreover, with $2 K \leq N$, the feedback controller,

$$
u=-\sum_{i=1}^{2 K}\left(R_{N} y, \psi_{i}\right)_{\omega} \psi_{i}
$$


once inserted in (14), exponentially stabilizes the corresponding closedloop system (14). The margin of stability for such closed loop system is $\lambda$. [See Remark 3.3.1 of [4] for the effective number of controls $2 K \leq N$.] More specifically, this means that the solution of

$$
\frac{d y}{d t}+\nu A y+A_{0} y+P\left(m \sum_{i=1}^{2 K}\left(R_{N} y, \psi_{i}\right)_{\omega} \psi_{i}\right)=0, y(0)=y^{0} \in \mathcal{D}\left(A^{\frac{1}{4}}\right)
$$

satisfies

$$
\left|A^{\frac{1}{4}} y(t)\right| \leq C_{\lambda} e^{-\lambda t}\left|A^{\frac{1}{4}} y^{0}\right|, t \geq 0 .
$$

Non-linear system (9). We next use the stabilizer in Theorem 1 to the linearized system (14) of the translated problem (10), or (11), to obtain the sought-after closed loop, local, feedback stabilization of the steady state solution $y_{e}$ to the N-S equation (9).

THEOREM 2 With reference to Theorem 1, the feedback controller

$$
u=-\sum_{i=1}^{2 K}\left(R_{N}\left(y-y_{e}\right), \psi_{i}\right)_{\omega} \psi_{i}
$$

[where the vectors $\psi_{i}$ are defined in (3.3.5) of Lemma 4 in [4]], once inserted in the $N$-S system (9) exponentially stabilizes the steady state solution $y_{e}$ to (1) in a neighborhood

$$
\mathcal{V}_{\rho}=\left\{y_{0} \in D\left(A^{\frac{1}{4}}\right) ;\left|A^{\frac{1}{4}}\left(y_{0}-y_{e}\right)\right|<\rho\right\}
$$

of $y_{e}$, for suitable $\rho>0$. More precisely, if $\rho>0$ is sufficiently small, then for each $y_{0} \in \mathcal{V}_{\rho}$ there exists a weak solution $y \in L^{\infty}(0, T ; H) \cap$ $L^{2}(0, T ; V)$, $\frac{d y}{d t} \in L^{\frac{4}{3}}\left(0, T ; V^{\prime}\right)$ for $d=3$, and $\frac{d y}{d t} \in L^{2}\left(0, T ; V^{\prime}\right)$ for $d=2, \forall T>0$, to the closed loop system

$$
\frac{d y}{d t}+\nu A y+B y+P\left(m \sum_{i=1}^{2 K}\left(R_{N}\left(y-y_{e}\right), \psi_{i}\right)_{\omega} \psi_{i}\right)=P f_{e}, t \geq 0, y(0)=y^{0}
$$

obtained from inserting the control (20) in (9), such that the following two properties hold:

(i)

$$
\int_{0}^{\infty} e^{2 \lambda t}\left|A^{\frac{3}{4}}\left(y(t)-y_{e}\right)\right|^{2} d t \leq C_{2}\left|A^{\frac{1}{4}}\left(y_{0}-y_{e}\right)\right|^{2} ;
$$

(ii)

$$
\left|A^{\frac{1}{4}}\left(y(t)-y_{e}\right)\right| \leq C_{3} e^{-\lambda t}\left|A^{\frac{1}{4}}\left(y_{0}-y_{e}\right)\right|, \quad \forall t \geq 0 .
$$


We refer to $[6$, p. 71$]$ for definition of weak solutions to equations of the form (22) and the asserted regularity. If $d=2$ the solution to (22) is strong and unique $[6, \mathrm{p} .83]$.

The pressure $p$. Theorem 2 implies the following result giving corresponding asymptotic properties of the pressure $p$.

THEOREM 3 The solution y provided by Theorem 2 satisfies also the equation

$$
\begin{aligned}
& y_{t}-\nu \Delta y+(y \cdot \nabla) y+m\left(\sum_{i=1}^{2 K}\left(R_{N}\left(y-y_{e}\right), \psi_{i}\right)_{\omega} \psi_{i}\right) \\
& =\nabla p+f_{e} \text { in } Q \equiv \Omega \times(0, \infty) ; \\
& \nabla \cdot y \equiv 0 \quad \text { in } Q \\
& y \equiv 0 \quad \text { on } \Sigma=\partial \Omega \times(0, \infty) \text {; } \\
& y(x, 0)=y_{0}(x) \text { in } \Omega \text {. }
\end{aligned}
$$

Moreover, the following relations hold true for the pressure $p$ :

(i) for $d=2$, we have

$$
\int_{0}^{\infty} t\left|p(t)-p_{e}\right|_{\left(H^{1}(\Omega)\right)^{d}}^{2} d t \leq C\left|A^{\frac{1}{4}}\left(y_{0}-y_{e}\right)\right|^{2}\left[1+\left|A^{\frac{1}{4}}\left(y_{0}-y_{e}\right)\right|^{2}\right]
$$

(ii) for $d=3$, we have

$$
\left.\int_{0}^{\infty}\left|p(t)-p_{e}\right|_{\left(L^{2}(\Omega)\right)^{d} / R^{2}}^{2} d t \leq C\left|A^{\frac{1}{4}}\left(y-y_{e}\right)\right|^{2}\right]\left[1+\left|A^{\frac{1}{4}}\left(y_{0}-y_{e}\right)\right|^{2}\right]
$$

\section{Part II: Boundary Control [3]}

\section{Introduction}

Boundary controlled Navier-Stokes equations. We consider the controlled Navier-Stokes equations (see [6, p. 45], [13, p. 253] for the uncontrolled case $u \equiv 0$ ) with boundary control $u$ in the Dirichlet B.C.:

$$
\begin{array}{rcc}
y_{t}(x, t)-\nu_{0} \Delta y(x, t)+(y \cdot \nabla) y(x, t) & =f_{e}(x) & \\
+\nabla p(x, t) & \text { in } G ; \\
\nabla \cdot y & =0 & \text { in } G ; \\
y & =u & \text { on } \Sigma ; \\
y(x, 0) & =y_{0}(x) & \text { in } \Omega .
\end{array}
$$


Here, $G=\Omega \times(0, \infty) ; \Sigma=\partial \Omega \times(0, \infty)$ and $\Omega$ is an open smooth bounded domain of $R^{d}, d=2,3 ; u \in L^{2}\left(0, T ;\left(L^{2}(\partial \Omega)\right)^{d}\right)$ is the boundary control input; and $y=\left(y_{1}, y_{2}, \ldots, y_{d}\right)$ is the state (velocity) of the system. The constant $\nu_{0}>0$ is the viscosity coefficient. The functions $y_{0}, f_{e} \in\left(L^{2}(\Omega)\right)^{d}$ are given, the latter being a body force, while $p$ is the unknown pressure. Because of the divergence theorem: $\int_{\Omega} \nabla \cdot y d \Omega=\int_{\Gamma} y \cdot \nu d \Gamma,[\Gamma=\partial \Omega, \nu=$ unit outward normal to $\partial \Omega]$, we must require (at least) the integral boundary compatibility condition: $\int_{\Gamma} u \cdot \nu d \Gamma=0$ on the control function $u$. Actually, a more stringent condition has to be imposed, in our final results: $u \cdot \nu \equiv 0$ on $\Sigma$, to sustain the pointwise boundary compatibility condition contained in the definition of the critical state space $H$ in (28e) below. To summarize we shall then assume

$$
\text { either } u \cdot \nu \equiv 0 \text { on } \Sigma \text {; or at least } \int_{\Gamma} u \cdot \nu d \Gamma \equiv 0 \text {, a.e. } t>0 \text {, }
$$

as it will be specified on a case-by-case basis.

\section{Steady-state solutions and space $V$ : sames as in (2), (3).}

Goal. Our goal is to construct a boundary control $u$, subject to the boundary compatibility condition (c.c.) given by (28e) in the strong pointwise form $u \cdot \nu \equiv 0$ on $\partial \Omega$, and, moreover, in feedback form $u=u(y)$ via some linear operator $y \rightarrow u$, such that, once $u(y)$ is substituted in the translated problem (28), the resulting well-posed, closed-loop system (28a) - (28c) possesses the following desirable property: the steady-state solutions $y_{e}$ defined in (2) are locally exponentially stable. In particular, motivated by our prior effort [4] to be described below, we seek to investigate if and when the feedback controller $u=u(y)$ can be chosen to be finite-dimensional, and, moreover, to act on an arbitrarily small portion (of positive measure) of the boundary $\Gamma=\partial \Omega$.

Orientation. Use of the Optimal Control Problem and Algebraic Riccati Theory. $(d=3)$ We emphasize here only the more demanding case of $d=3$. A preliminary difficulty (for $d=2,3$ ) is the requirement in (28e) that the boundary control $u$ must always be tangential at each point of the boundary. It is standard that this requirement is intrinsically built in the definition of the state space $H$ (above in (5) Part I) of the velocity vectory $y$, which is critical to eliminate the second unknown of the N-S model, the pressure term $\nabla p$ (see the orthogonal complement $H^{\perp}$ in (5) above, Part I), by virtue of the Leray projection $P$. Evolution of the velocity must occur in $H$. Accordingly, we must then have that the boundary controls be pointwise tangential: $u \cdot \nu \equiv 0$ on $\Sigma$ in (28e). Next, a second difficulty, this time for $d=3$, 
is that the non-linearity of the N-S equation dictates and forces the requirement that stabilization must occur in the space $\left(H^{\frac{3}{2}+\epsilon}(\Omega)\right)^{3}, \epsilon>0$, see Eqn. (5.18a-b) of [3]. This is a high topological level, of which we shall have to say more below. A third source of difficulty consists in deciding how to inject 'dissipation' into the N-S model, in fact, as required, through a boundary tangential controller expressed in feedback form. Here, motivated by [4] and, in turn, by optimal control theory [12], in order to inject dissipation into the N-S system as to force local exponential boundary stabilization of its steady-state solutions, we choose the strategy of introducing an Optimal Control Problem (OCP) with a quadratic cost functional, over an infinite time-horizon, for the linearized N-S model subject to tangential Dirichlet-boundary control $u$, i.e., satisfying $u \cdot \nu \equiv 0$ on $\Sigma$. One then seeks to express the boundary feedback, closed-loop controller of the optimal solution of the OCP, in terms of the Riccati operator arising in the corresponding algebraic Riccati theory. As a result, the same Riccati-based boundary feedback optimal controller that is obtained in the linearized OCP is then selected and implemented also on the full N-S system. This controller in feedback form is both dissipative as well as 'robust' (with respect to a certain class of perturbations). For $d=3$, however, the OCP must be resolved at the high $\left(H^{\frac{3}{2}+\epsilon}(\Omega)\right)^{3}$-topological level, within the class of Dirichlet boundary controls in $L^{2}\left(0, \infty ;(\partial \Omega)^{3}\right)$, which are further constrained to be tangential to the boundary.

Thus, the OCP faces two additional difficulties that set it apart and definitely outside the boundaries of established optimal control theory for parabolic systems with boundary controls: (1) the high degree of unboundedness of the boundary control operator, of order $\left(\frac{3}{4}+\epsilon\right)$ as expressed in terms of fractional powers of the basic free-dynamics generator; and (2) the high degree of unboundedness of the 'penalization' or 'observation' operator of order also $\left(\frac{3}{4}+\epsilon\right)$, as expressed in terms of fractional powers of the basic free-dynamics generator. This yields a 'combined index' of unboundedness strictly greater than $\frac{3}{2}$. By contrast, the established (and rich) optimal control theory of boundary control parabolic problems and corresponding algebraic Riccati theory requires a 'combined index' of unboundedness strictly less than 1 [12, Vol. 1, in particular, p. 501-503], which is the maximum limit handled by perturbation theory of analytic semigroups. To implement this program, however, one must first overcome, at the very outset, the preliminary stumbling obstacle of showing that the present highly non-standard OCP - with the aforementioned high level of combined unboundedness in control and observation operators and further restricted within the class of tangential boundary controllers-is, in fact, non-empty. This 
result is achieved in Theorem 3.5.1 of [3] in full generality (and in Proposition 3.7.1 of [3] under the assumption that the linearized operator is diagonalizable over the finite-dimensional unstable subspace). Thus, after this result, the study of the OCP may then begin. Because of the aforementioned intrinsic difficulties of the OCP with a combined index of unboundedness $>\frac{3}{2}$, one cannot (and cannot hope to) recover in full all desirable features of the corresponding algebraic Riccati theory which are available when the combined index of unboundedness in control and observation operators is strictly less than 1 ([12] and references therein). For instance, existence of a solution (Riccati operator) of the algebraic Riccati equation is here asserted only on the domain of the generator of the optimal feedback dynamics (Proposition 4.5.1 of [3]); not on the domain of the free-dynamics operator, as it would be required by, or at least desirable from, the viewpoint of the OCP. However, in our present treatment, the OCP is a means to extract dissipation and stability, not an end in itself. And indeed, the present study of the algebraic Riccati theory, with a combined index of unboundedness in control and observation operator strictly above $\frac{3}{2}$ (rather than strictly less than 1) does manage, at the end, to draw out the key sought-after features of interest--dissipativity and decay-for the resulting optimal solution in feedback form of the OCP for the linearized N-S equation. All this is accomplished in Section 4 of [3].

The subsequent step of the strategy is then to select and use the same Riccati-based, boundary feedback operator, which was found to describe the optimal solution of OCP of the linearized N-S equation, directly into the full N-S model. For $d=3$, the heavy groundwork for the feedback stabilization of the linearized problem via optimal control theory makes then the resulting analysis of well-posedness (in Section 5 of [3]) and stabilization (in Section 6) of the N-S model more amenable than would otherwise be the case.

To this end, key use is made of the Algebraic Riccati Equation satisfied by the Riccati operator that describes the stabilizing control in closedloop feedback form.

Literature. This paper [3]is a successor to [4], which instead considered the interior stabilization problem of the Navier-Stokes equations, that is, problem (1), Part I, with (i) non-slip boundary condition $y \equiv 0$ on $\Sigma \equiv \partial \Omega \times(0, \infty)$ in place of the boundary controlled condition (28b); and (ii) interior control $m(x) u(x, t)$ on the right-hand side of Eqn. (28a), where $m(x)$ is the characteristic function of an arbitrary open subset $\omega \subset \Omega$ of positive measure. In this case [4] (Part I) proves that (the linearized problem is exponentially stabilizable, hence that) the steady-state solutions $y_{e}$ to the Navier-Stokes equations are locally 
exponentially stabilizable by a finite-dimensional feedback controller, in fact, of minimal size, see Part I. In addition, one may select the finitedimensional feedback controller to be expressed in terms of a Riccati operator (solution of an algebraic Riccati equation, which arises in an optimization problem associated with the linearized equation). We shall need to invoke this interior stabilization problem (though not in its full strength) in Section 3.5 of [3].

The work in the literature which is most relevant to our present paper is that of A. Fursikov, see [8] (of which we become aware after completing [4]), which culminates a series of papers quoted therein. A statement of the main contribution of [8], as it pertains to the linearized problem (31) below, is contained in [8, Theorems 3.3 and 3.5, pp. 104-5]. Given the pair $\left\{\Omega, \Gamma_{0}\right\}$, where $\Gamma_{0}$ is a portion of the boundary $\partial \Omega$ of $\Omega$, the approach of [8] starts with a special class, called $V^{1}\left(\Omega, \Gamma_{0}\right)$, of initial conditions $y^{0}$ for the linearized problem (31) defined on $\Omega$. More specifically, $y^{0} \in$ $V^{1}\left(\Omega, \Gamma_{0}\right)$ means that the initial vector $y^{0}$ is the restriction $y^{0}=\left.Y^{0}\right|_{\Omega}$ on $\Omega$ of the vector $Y^{0}$ on the extended domain $G=\Omega \cup \omega$, where: (i) $y^{0} \in\left(H^{1}(G)\right)^{d}, d=2,3$; (ii) $\nabla \cdot Y^{0} \equiv 0$ in $G$; (iii) $\left.Y^{0}\right|_{\partial G}=0$; (iv) the set $\omega$ is an estension of $\Omega$ across the pre-assigned portion $\Gamma_{0}$ of $\partial \Omega$. Then, [8, Theorem 3.3] establishes that such $y^{0} \in V^{1}\left(\Omega, \Gamma_{0}\right)$ can, in turn, be re-extended from $\Omega$ to $G$ as a new vector $\eta^{0} \in\left(H^{1}(G)\right)^{d}$, $\nabla \cdot \eta^{0} \equiv 0$ in $G,\left.\eta^{0}\right|_{\partial G}=0$, possessing now the new critical property that, in addition, $\eta^{0}$ belongs to the stable subspace of the linearized operator $\tilde{\mathcal{A}}$. Here $\tilde{\mathcal{A}}$ is an extension of the original operator $\mathcal{A}$ in (6) Part I from $\Omega$ to $G$, obtained by a corresponding extension of the steady-state solution $y_{e}$ from $\Omega$ to $G$, while preserving the required properties of being divergence-free across $G$ and vanishing on $\partial G$. As a consequence of this extension, one obtains a function $\eta\left(t ; \eta^{0}\right)=e^{\tilde{\mathcal{A}} t} \eta^{0}$, which is the solution of the corresponding linearized problem, except this time on $G$, and with homogeneous Dirichlet (non-slip) boundary condition on $\partial G$. Then, [8, Theorem 3.5] concludes (because of the obvious invariance of the stable subspace for the s.c. analytic semigroup $\left.e^{\tilde{\mathcal{A}} t}\right)$ that such $\eta\left(t ; \eta^{0}\right)$ is exponentially decaying: $\left\|e^{\tilde{\mathcal{A}} t} \eta^{0}\right\| \leq C e^{-\sigma t}\left\|\eta^{0}\right\|$, in the $\left(H^{1}(G)\right)^{d}$-norm \|\| , with a controlled decay rate $\sigma, 0<\sigma<\left|\operatorname{Re} \lambda_{N+1}\right|$. At this point, [8] takes the restrictions of $\eta\left(t ; \eta^{0}\right)$ on $\Omega$ and $\Gamma_{0}: y(t)=\left.\eta\left(t ; \eta^{0}\right)\right|_{\Omega}$, $u(t)=\left.\eta\left(t ; \eta^{0}\right)\right|_{\Gamma_{0}}$, with $u(t) \equiv 0$ on $\Gamma \backslash \Gamma_{0}$, and defines such $u$ as a "feedback control" of the corresponding solution $y$ of problem (31) below on $\Omega$, which then stabilizes such solution $y(t)$ of $(31)$ below over $\Omega$. A similar definition of "feedback control" is given in [8] with respect to the non-linear Navier-Stokes problem. 
One should note, however, that the aforementioned controller for problem (31) below given in [8] is not a feedback controller in the standard sense. Instead, our main results (in [4] as well as) in the present paper construct genuine, authentic, and real feedback controls (Riccatibased, in fact, hence with some feature of 'robustness'), that use at time $t$ only the state information on $\Omega$ at time $t$. The present paper, therefore, encounters a host of technical problems not present in [8]: from the need for the genuine feedback control $u$ to satisfy the pointwise compatibility condition $u \cdot \nu \equiv 0$ on $\Sigma$; to the high topological level $\left(H^{\frac{3}{2}+\epsilon}(\Omega)\right)^{3}$ at which stabilization must occur in our case, as dictated by the non-linearity for $d=3$, see Eqn. $(5.18 \mathrm{a}-\mathrm{b})$ of [3], versus the $H^{1}$ topology decay obtained in [8]; to the treatment of the Riccati theory for a corresponding optimal control problem with a combined 'index of unboundedness' in control and observation operators exceeding $\frac{3}{2}$-thus $\frac{1}{2}+2 \epsilon$ beyond the (rich) theory of the literature [12], as explained in the Orientation.

Main contributions of the present paper. Qualitative summary of main results. A first qualitative description of the main results of the present paper follows next. First of all, the pre-set goal is achieved: with no assumptions whatsoever (except mild assumptions on the domain), we prove here that the steady-state solutions to NavierStokes equations on $\Omega \subset \mathbb{R}^{d}, d=2,3$, are locally exponentially stabilizable by a closed-loop boundary feedback controller acting in the Dirichlet boundary conditions in the required topologies [Theorem 2.3 for $d=3$ and Theorem 2.6 for $d=2$ of [3]]. The feedback controller is expressed in terms of a Riccati operator (solution of a suitable algebraic Riccati equation): as such, via standard arguments (e.g., [4]) this feedback controller is 'robust' with respect to a certain class of exogeneous perturbations.

More precisely, the following main results are established in the present paper:

(i) For the general cases $d=2,3$, an infinite-dimensional, closed-loop boundary feedback, stabilizing controller is constructed, as acting (for general initial data) on the entire boundary $\partial \Omega$ for $d=3$; or on an arbitrarily small portion of the boundary, for $d=2$.

(ii) By contrast, for $d=2$ and under a finite-dimensional spectral assumption FDSA = (3.6.2) of [3] (diagonalizability of the restriction of the linearized operator over the finite-dimensional unstable subspace), the feedback controller can be chosen to be finite-dimensional, with dimension related to properties of the unstable eigenvalues, and, moreover, still to act on an arbitrarily small portion of the boundary.

(iii) For $d=3$, local exponential feedback stabilization of the steadystate solutions to Navier-Stokes equations is not possible with a finite- 
dimensional boundary feedback controller (except for a meager set of special initial conditions).

(iv) The pathology noted in (iii) for $d=3$ is due to the non-linearity (see Eqn. (5.18a-b) of [3]) which (by Sobolev embedding and multiplier theory for $d=3$ ) forces the the requirement that solutions of the linearized problem be considered at the high regularity space $H^{\frac{3}{2}+\epsilon}(\Omega) \cap H$, $\epsilon>0$, under initial conditions $y_{0} \in H^{\frac{1}{2}+\epsilon}(\Omega) \cap H$ and $L^{2}\left(0, \infty ;\left(L^{2}(\Gamma)\right)^{d}\right)$ boundary controls $u$. In turn, this high regularity space $H^{\frac{3}{2}+\epsilon}(\Omega)$ causes the occurrence of the compatibility condition $\left.y_{0}\right|_{\Gamma}=u(0)$ at $t=0$ on the boundary to be satisfied. Thus, for $d=3$, the constructed feedback controller must be infinite-dimensional in general.

(v) By contrast, the linearized problem for $d=2,3$ is exponentially stabilizable with a closed-loop boundary, finite-dimensional feedbackcontroller acting on an arbitrarily small portion of the boundary up to the topological level $\left(H^{\frac{3}{2}-\epsilon}(\Omega)\right)^{d}$ and with initial conditions $y_{0} \in$ $\left(H^{\frac{1}{2}-\epsilon}(\Omega)\right)^{d} \cap H$, under the same FDSA $=(3.6 .2)$ of [3].

Notation and preliminaries. Same as in Part I.

\section{Main Results (Case $d=3$ )}

The following assumptions will be in effect throughout the paper.

Assumptions. (i) The boundary $\partial \Omega$ of $\Omega$ is a finite union of $d-1$ dimensional $C^{2}$-connected manifolds.

(ii) The steady-state solution $\left(y_{e}, p_{e}\right)$ defined in (2) Part I, belongs to $\left(\left(H^{2}(\Omega)\right)^{d} \cap V\right) \times H^{1}(\Omega)$. [For $d=2,3$, this property is guaranteed by $\left[6\right.$, Theorem 7.3 , p. 59] on $y_{e}$, for $f_{e} \in H$, followed by [6, Theorem 3.11, p. 30] on $p_{e}$, for sufficiently smooth $\partial \Omega$.]

Preliminaries. The translated non-linear N-S problem. By the substitutions $y \rightarrow y_{e}+y, p \rightarrow p_{e}+p$ and $\left.u \rightarrow y_{e}\right|_{\Gamma}+u\left(\left.y_{e}\right|_{\Gamma}\right.$ being the Dirichlet trace of $y_{e}$ on $\Gamma \equiv \partial \Omega$ ), we are readily led via (28), (2) to study the boundary null stabilization of the equation

$$
\begin{aligned}
& y_{t}-\nu_{0} \Delta y+(y \cdot \nabla) y+\left(y_{e} \cdot \nabla\right) y \\
& +(y \cdot \nabla) y_{e}=\quad \nabla p \quad \text { in } Q ; \\
& \nabla \cdot y=\quad 0 \quad \text { in } Q \\
& y=\quad u \quad \text { on } \Sigma \text {; } \\
& y(x, 0)=y^{0}(x)=y_{0}(x)-y_{e}(x) \text { in } \Omega .
\end{aligned}
$$

Abstract model of the N-S problem (29) projected on $H$. We shall see in Section 3.1 of [3] that, under the pointwise compatibility 
condition (c.c.) $u \cdot \nu=0$ on $\Sigma$ of $(28 \mathrm{e})$ (whereby then $P y_{t}=y_{t}$ ), application of the Leray projection $P$ on $(29 \mathrm{a})-(29 \mathrm{~d})$ leads to a corresponding equation in $H$, without the pressure terms, whose abstract version can be written as

$$
y_{t}-\mathcal{A} y+B y=-\mathcal{A} D u, \quad y(0)=y^{0} \in H, \quad u \cdot \nu \equiv 0 \text { on } \Sigma,
$$

where the infinitesimal generator $\mathcal{A}$ and the non-linear operator $B$ are defined in (15) and (7), respectively, of Part I. Moreover, the operator $D:\left(L^{2}(\Gamma)\right)^{d} \rightarrow\left(H^{\frac{1}{2}}(\Omega)\right)^{d} \cap H \in \mathcal{D}\left(A^{\frac{1}{4}-\epsilon}\right)$ is defined in (3.1.3) of [3].

The translated linearized problem. PDE version. The translated linearized problem corresponding to (29) is then

$$
\begin{aligned}
y_{t}-\nu_{0} \Delta y+\left(y_{e} \cdot \nabla\right) y+(y \cdot \nabla) y_{e} & =\nabla p & & \text { in } Q ; \\
\nabla \cdot y & =0 & & \text { in } Q ; \\
y & =u & & \text { on } \Sigma ; \\
y(x, 0) & =y^{0}(x) & & \text { in } \Omega .
\end{aligned}
$$

Abstract model of problem (31) projected on $H$. Its abstract version on $H$ is then

$$
y_{t}=\mathcal{A} y-\mathcal{A} D u \in\left[\mathcal{D}\left(\mathcal{A}^{*}\right)\right]^{\prime}, \quad y(0)=y^{0} \in H ; \quad u \cdot \nu \equiv \text { on } \Sigma .
$$

Main results: Case $d=3$. The linearized model. We begin with the translated linearized problem (31) or its projected version (32). For the first result--the main result on problem (32) - essentially no assumptions are required.

TheOREM 4 With reference to the linearized problem (32), Part II, the following results hold true:

(i) Let $d=3$ and assume further that $\Omega$ is simply connected. Then, given any $y^{0} \in W \equiv\left(H^{\frac{1}{2}+\epsilon}(\Omega)\right)^{3} \cap H, \epsilon>0$ arbitrary, there exists an open-loop, infinite-dimensional boundary control $u \in L^{2}\left(0, \infty ;\left(L^{2}(\Gamma)\right)^{3}\right)$, $u \cdot \nu \equiv 0$ on $\Sigma$, such that the corresponding solution $y$ of (32) satisfies $y \in$ $L^{2}\left(0, \infty ;\left(H^{\frac{3}{2}+\epsilon}(\Omega)\right)^{3} \cap H\right) \cap H^{\frac{3}{4}+\frac{\epsilon}{2}}(0, \infty ; H)$. Moreover, if $y^{0}$ vanishes on the portion $\Gamma_{0}$ of the boundary $\Gamma=\partial \Omega$, then $u$ may be required to act only on the comlementary part $\Gamma_{1}=\Gamma \backslash \Gamma_{0}$ of the boundary. In particular, if $y^{0}$ vanishes on all of $\Gamma$, then $u$ may be required to have an arbitrarily small support $\Gamma_{1}$, meas $\left(\Gamma_{1}\right)>0$. [This is Theorem 3.5.1 along with Remark 3.5.1, illustrated by Figures 3.5.1 and 3.5.2 in [3].] 
(ii) Let $d=3$. Then, the control u claimed in (i) cannot generally be finite-dimensional except for a meager set of special initial conditions. [This is Proposition 3.1.3 of [3].]

Case $d=3$. Original N-S model (28). We now report the main result of the present paper, which provides the sought-after closed-loop boundary feedback control for the original N-S equations (28) [or its projected version (30)], which exponentially stabilizes the stationary solution $y_{e}$ of (28) in a neighborhood of $y_{e}$. The stabilizing feedback control that we shall find is 'robust,' as it is expressed in terms of a Riccati operator $R$, which arises in an associated corresponding Optimal Control Problem. To state our (local) stabilizing result, we need to introduce the set

$$
\mathcal{V}_{\rho} \equiv\left\{y_{0} \in W \equiv\left(H^{\frac{1}{2}+\epsilon}(\Omega)\right)^{3} \cap H:\left|y_{0}-y_{e}\right|_{W}<\rho\right\}
$$

of initial conditions $y_{0}$ of (28), whose distance in the norm of $W$ from a stationary solution $y_{e}$ is less than $\rho>0$. Here, $\epsilon>0$ arbitrary is fixed once and for all.

THEOREM 5 (MAIN THEOREM) Let $d=3$ and assume further that $\Omega$ is simply connected. If $\rho>0$ in (33) is sufficiently small, then: for each $y_{0} \in \mathcal{V}_{\rho}$, there exists a unique fixed-point, mild, semigroup solution $y$ of the following closed-loop problem:

$$
\begin{array}{r}
y_{t}(x, t)-\nu_{0} \Delta y(x, t)+(y \cdot \nabla) y(x, t) \\
=f_{e}(x)+\nabla p(x, t) \text { in } G ; \\
\nabla \cdot y=0 \text { in } G ; \\
y=\nu_{0} \frac{\partial}{\partial \nu} R\left(y-y_{e}\right) \text { on } \Sigma ; \\
y(x, 0)=y_{0}(x) \text { in } \Omega .
\end{array}
$$

obtained from (28) by replacing $u$ with the boundary feedback control $u=y_{e}+\nu_{0} \frac{\partial}{\partial \nu} R\left(y-y_{e}\right)$ having the following regularity and asymptotic properties:

(i)

$$
\left(y-y_{e}\right) \in C([0, \infty) ; W) \cap L^{2}\left(0, \infty ;\left(H^{\frac{3}{2}+\epsilon}(\Omega)\right)^{3} \cap H\right)
$$

continuously in $y_{0} \in W \equiv\left(H^{\frac{1}{2}+\epsilon}(\Omega)\right)^{3} \cap H$ :

$$
\left|y(t)-y_{e}\right|_{W}^{2}+\int_{0}^{\infty}\left|y(t)-y_{e}\right|_{\left(H^{\frac{3}{2}+\epsilon}(\Omega)\right)^{3} \cap H}^{2} d t \leq C\left|y_{0}-y_{e}\right|_{W}^{2}, t \geq 0 .
$$


[This follows from Theorem 5.1 and Corollary 5.5 of [3], via the translation $y \rightarrow y_{e}$, etc., performed above problem (29).]

(ii) there exist constants $M \geq 1, \omega>0$ (independent of $\rho>0$ ) such that such solution $y(t)$ satisfies

$$
\left|y(t)-y_{e}\right|_{W} \leq M e^{-\omega t}\left|y_{0}-y_{e}\right|_{W}, \quad t \geq 0 .
$$

[This follows from Theorem 6.1(i) of [3], via the translation $y \rightarrow y-y_{e}$, etc., performed above problem (29).]

Here $R$ is a Riccati operator, in the sense that it [arises in the Optimal Control Problem of Section 4.1 and] satisfies the Algebraic Riccati Equation (4.5.1) of [3]. The operator $R$ is positive self-adjoint on $H$ and, moreover, $R \in \mathcal{L}\left(W ; W^{\prime}\right)$ where $W^{\prime}$ is the dual of $W$ with respect to $H$ as a pivot space. In addition [Proposition 4.1.4 of [3]],

$$
c|x|_{W}^{2} \leq(R x, x)_{H} \leq C|x|_{W}^{2}, 0<c<C<\infty, \forall x \in W
$$

so that the $\left|R^{\frac{1}{2}} x\right|$-norm is equivalent to the $W$-norm. By a solution to Eqn. (5)), we mean, of course, a weak solution (see, e.g., [6], [13]). (This part is Theorem 5.1 of [3].)

\section{References}

[1] V. Barbu. Local internal controllability of the Navier-Stokes equations. Advances in Differential Equations, 6(12):1443-1462, 2001.

[2] V. Barbu. Feedback stabilization of Navier-Stokes equations. ESAIM COCV, 9:197-206, 2003.

[3] V. Barbu, I. Lasiecka, and R. Triggiani. Boundary stabilization of Navier-Stokes equations. 2003. Preprint.

[4] V. Barbu and R. Triggiani. Internal stabilization of Navier-Stokes equations with finite-dimensional control. Indiana University Mathematics Journal, 2004. To appear.

[5] V. Barbu and G. Wang. Feedback stabilization of the semilinear heat equations. Abstract and Applied Analysis, 12:697-714, 2003.

[6] P. Constantin and C. Foias. Navier-Stokes Equations. University of Chicago Press, Chicago, London, 1988.

[7] J. M. Coron. On the null asymptotic stabilization of the 2-d incompressible Euler equations in a simply connected domain. SIAM J. Control Optimiz., 37:1874-1896, 1999 .

[8] A. Fursikov. Real Process Corresponding to the $3 D$ Navier-Stokes System and its Feedback Stabilization from the Boundary, volume 206 of Translations of the AMS, Series 2. American Mathematical Society, 2002. Partial Differential Equations, Mark Vishik's Seminar, pp. 95-123, M. S. Agranovich and M. A. Shubin, editors. 
[9] O. A. Imanuvilov. Local controllability of Navier-Stokes equations. ESAIM COCV, 3:97-131, 1998. On local controllability of Navier-Stokes equations, ESAIM COCV 6 (2001), 49-97.

[10] T. Kato. Perturbation Theory of Linear Operators. Springer-Verlag, New York, Berlin, 1966.

[11] I. Lasiecka. Exponential stabilization of hyperbolic systems with nonlinear unbounded perturbations-a riccati operator approach. Applicable Analysis, 42:243-261, 1991.

[12] I. Lasiecka and R. Triggiani. Control Theory for Partial Differential Equations: Continuous and Approximation Theories, volume 1 of Encyclopedia of Mathematics and its Applications. Cambridge University Press, 2000. 644 pp.

[13] R. Teman. Navier-Stokes Equations, Revised edition, volume 2 of Studies in Mathematics and its Applications. North Holland, 1979.

[14] R. Triggiani. On the stabilizability problem in Banach space. J. Math. Anal. Appl., 52:383-403, 1975.

[15] R. Triggiani. Boundary feedback stabilizability of parabolic equations. Appl. Math. Optimiz., 6:201-220, 1980. 